\title{
Johann Rists Monatliche Unterredungen als rhetorische Konversationsanleitung
}

\author{
Michael Multhammer
}

Online publiziert: 5. Februar 2020

(C) Der/die Autor(en) 2020

Zusammenfassung Der Beitrag stellt die Form von Johann Rists Monatlichen Unterredungen in den Fokus. Dabei soll erwiesen werden, dass die dort zum Tragen kommenden rhetorischen Strukturen der eigentliche Endzweck der Schriften sind, wodurch sie anhand der Besprechung aktueller Inhalte zu einer umfassenden Rhetorikschulung für die zeitgenössische Jugend werden. Im Anschluss an Habermas wird über den Begriff einer >Konversationsöffentlichkeit< und dessen Potenzial zur Klärung von Gattungsfragen reflektiert.

Schlüsselwörter Johann Rist · Konversation · Rhetorik · Gespräch · Barock · Öffentlichkeit

\section{Johann Rist's Monatliche Unterredungen as a Rhetorical Introduction to the Art of Conversation}

\begin{abstract}
The article focuses on the form of Johann Rist's Monatlichen Unterredungen. The aim is to prove that the rhetorical structures used there are the actual final purpose of these writings, making them a comprehensive training in rhetorical strategies for the contemporary youth by discussing current content. Following Habermas, the term »Konversationsöffentlichkeit « and its potential for clarifying generic questions are reflected on.
\end{abstract}

Keywords Johann Rist - Conversation · Rhetoric · Baroque · Public Sphere

M. Multhammer ( $\triangle)$

Germanistisches Seminar, Universität Siegen, Siegen, Deutschland

E-Mail: multhammer@germanistik.uni-siegen.de 


\section{Einleitung - Forschung}

Als der Barockdichter Johann Rist (1607-1667) ${ }^{1}$ im 18. Jahrhundert wiederentdeckt wird, sind es seine weltlichen und geistlichen Lieder, die alle Aufmerksamkeit auf sich ziehen. Diese Kanonisierung als dichtender Theologe, der allen voran für die Erbauung und den Gottesdienst erfolgreiche Kirchenlieder geschaffen hatte, ${ }^{2}$ fand über den Umweg der Volksdichtung statt, zu der Rists Werk nun - mehr als hundert Jahre nach seinem Ableben - gezählt werden konnte. ${ }^{3}$ Demgegenüber sind seine übrigen Dichtungen weitestgehend in Vergessenheit geraten, das trifft auf seine dramatischen Versuche ${ }^{4}$ ebenso zu wie auf das gelehrte Werk und Rists späte Epik. ${ }^{5}$ Das gilt auch lange Zeit für Rists Monatliche Unterredungen ${ }^{6}$, die erst in den letzten Jahren wieder - aus ganz unterschiedlichen Perspektiven - in den Blick der Forschung gekommen sind. Bis dahin galt, was schon Alfred Jericke 1928 konstatierte:

1 Zu Biographie und Werk siehe überblicksartig Mannack, Eberhard/Steiger, Johann Anselm: [Art.] »Rist,
Johann«. In: Killy Literaturlexikon. Hrsg. v. Wilhelm Kühlmann u. a. 2. vollständig überarbeitete Auflage.
13 Bde. Berlin 2008-2012. Hier Bd. 9, S. 668-670. Die umfassendste monographische Darstellung ist nach
wie vor Theodor Hansen: Johann Rist und seine Zeit. Halle 1872. Neudr. 1972. Für neuere Forschungen,
die einen Querschnitt durch Rists Oeuvre bieten, siehe jetzt den Sammelband Steiger, Johann Anselm/Jahn,
Bernhard (Hg.): Johann Rist (1607-1667). Profil und Netzwerke eines Pastors, Dichters und Gelehrten. Berlin, Boston 2015.

2 Zu Rists Schaffen als Lyriker siehe Mews, Rudolf: Johann Rists Gesellschaftslyrik und ihre Beziehung zur zeitgenössischen Poetik. Diss. Univ. Hamburg 1969; Moerke, Ulrich: Die Anfänge der weltlichen Barocklyrik in Schleswig-Holstein. Hudemann, Rist, Lund. Neumünster 1972; Scheitler, Irmgard: Das geistliche Lied im deutschen Barock. Berlin 1982; Marigold, W. Gordon: »Aspekte des geistlichen Liedes im 17. Jahrhundert. Johann Rist, Hinrich Elmenhorst, Christian Knorr von Rosenroth«. In: Morgen-Glantz 6 (1996), S. 81-98; Garber, Klaus: »Pétrarquisme pastoral et bourgeoisie protestante: La Poésie pastorale des Johann Rist et Jakob Schwieger«. In: Claude Longeon (Hg.): Le genre pastoral en Europe du XV au XVII ${ }^{e}$ siècle. Saint-Etienne 1980, S. 269-297; Krummacher, Hans-Henrik: »Lehr- und trostreiche Lieder. Johann Rists geistliche Dichtung und die Predigt- und Erbauungsliteratur des 16. und 17. Jahrhunderts«. In: Wolfgang Haubrich/Wolfgang Kleiber/Rudolf Voß (Hg.): Vox Sermo Res. Beiträge zur Sprachreflexion, Literatur- und Sprachgeschichte vom Mittelalter bis zur Neuzeit. FS für Uwe Ruberberg. Stuttgart 2001, S. 143-168.

3 Für diese Einordnung ist in erster Instanz Johann Gottfried Herder verantwortlich. Dieser nahm einige von Rists Gedichten in seine anonym erschienene Sammlung von Volksliedern auf. Die Anthologie sollte fernerhin zum Vorbild vieler weiterer werden. [Johan Gottfried Herder:] Volkslieder. Nebst untermischten andern Stücken. Zweiter Theil. Leipzig 1779.

4 Mannack, Eberhard: »Johann Rists Perseus und das Drama des Barock«. In: Daphnis 1 (1972), S. 141-149; Taylor, Irmgard C.: »Untersuchungen zum Stil der Dramen Johann Rists«. In: Korrespondenzblatt des Vereins für niederdeutsche Sprachforschung 79 (1972), H. 3, S. 33-36; Schiewek, Ingrid: »Theater zwischen Tradition und Neubeginn. Die Zwischenspiele des Johann Rist«. In: Werner Lenk (Hg.): Studien zur deutschen Literatur im 17. Jahrhundert. Berlin 1984, S. 145-251; Rahn, Thomas: »Krieg als Störfall der Rhetorik. Die Friedensspiele von Johann Rist und Justus Georg Schottelius«. In: Ders.: (Hg.): Krieg und Rhetorik. Tübingen 2003, S. 43-57.

5 Die entsprechende Literatur zur Epik wird im Verlauf der Abhandlung herangezogen.

6 Die Titel, die im Verlauf von vier Jahren erscheinen, lauten in verkürzter Form: Das alleredelste Nass der Welt; Das alleredelste Leben; Die Alleredelste Torheit; Die Alleredelste Belustigung kunst- und tugendliebender Gemüter; Die alleredelste Erfindung sowie Die alleredelste Zeitverkürzung. Zitiert wird im Folgenden nach der Ausgabe Rist, Johann: Sämtliche Werke, Bd. 4-6: Epische Dichtungen. Hg. v. Eberhard Mannack. Berlin 1972. 
Die gelegentlich sich findenden flüchtigen Urteile über diese Werke sind auffällig unbestimmt gehalten und machen den Eindruck, als wenn man nicht recht gewußt hätte, ob man die Schriften überhaupt in den Rahmen der Literaturgeschichte einfügen, und in welcher Gattung man sie dann unterbringen sollte. ${ }^{7}$

Diese Probleme haben sich nicht erledigt, zwar wird man heutzutage nur schwerlich abstreiten, dass Rists Monatsgespräche einen Platz in der deutschen Literaturgeschichte verdienen, wo dieser indes zu lokalisieren sei, hängt ganz dezidiert vom Blickwinkel ab. ${ }^{8}$ So galt etwa Nikola Kaminskis Interesse jüngst der avisierten periodischen Erscheinungsform der >Monatlichen Unterredungen $<$ und den damit entstehenden Schwierigkeiten in der Produktion eben jener. ${ }^{9}$ Die Frage, die im Hintergrund steht, ist, ob es sich bei Rists Monatsgesprächen in irgendeiner Form um einen Vorläufer der späterhin so erfolgreichen und wirkmächtigen Zeitschriftenliteratur handelt. Stellt man das naturkundliche und mechanische Wissen in den Mittelpunkt (Ferdinand van Ingen),${ }^{10}$ nähert man die Schriften gattungsgeschichtlich populärwissenschaftlichen Traktaten an. Ebenso ist eine inhaltliche Ausrichtung hin auf die umfassend behandelte Blumenkunde möglich (und auch sinnvoll). ${ }^{11}$ In der Verbindung mit Diskursen zur Gartenkultur werden zudem theologische Fragestellungen sichtbar. ${ }^{12}$ Darüber hinaus rückten jüngst die von Rist angestellten ästhetischen und dichtungstheoretischen Überlegungen in den Vorreden und eigentlichen Gesprächen - allen voran zur Paragone delle arti und Fragen das Theater betreffend - in den Fokus. ${ }^{13}$ All diesen Annäherungen an Rists späte epische Schriften ist eines gemein: Sie stellen vornehmlich inhaltliche Aspekte, die dort reichlich und auch

\footnotetext{
7 Jericke, Alfred: Johann Rists Monatsgespräche. Berlin, Leipzig 1928, S. 2.

8 Eine mögliche Einordnung findet sich bei Stockhorst, Stefanie: Reformpoetik. Kodifizierte Genustheorie des Barock und alternative Normenbildung in poetologischen Paratexten. Tübingen 2008, S. 399-404.

9 Kaminski, Nikola: »>Monatliche Unterredungen, ist ein Journal<. Rists >Jänners< bis >Brachmonats $<-$ Unterredungen im Horizont von Zeitschriften-/Fortsetzungsliteratur«. In: Johann Rist (1607-1667). Profil und Netzwerke eines Pastors, Dichters und Gelehrten. Hg. v. Johann Anselm Steiger u. Bernhard Jahn. Berlin, Boston 2015, S. 587-610.

10 Ingen, Ferdinand van: »Johann Rist und die Naturwissenschaften seiner Zeit. Anmerkungen zu seiner intellektuellen Biographie anlässlich des 400. Geburtstages am 8.3.2007«. In: Daphnis 36 (2007), S. 487-510.

11 Jörg Wesche spricht ganz treffend von einer »florilistische[n] lectio«. Wesche, Jörg: »Floriographie bei Rist«. In: Johann Rist (1607-1667). Profil und Netzwerke eines Pastors, Dichters und Gelehrten. Hg. v. Johann Anselm Steiger u. Bernhard Jahn. Berlin, Boston 2015, S. 655-672, hier S. 660. Ferner Linnemann, Johanna-Helene: »>Bluhmen-Discurse< in Johann Rists >Jahreszeitengespräche<: Figuren, Texte und epistemische Ordnungen im Dialog «. In: Carmen Ulrich (Hg): Dialog und Dialogizität: interdisziplinär, interkulturell, international. München 2017, S. 145-159.

12 Stockhorst, Stefanie: »Wissensvermittlung im Dialog. Literarische Pflanzenkunde und christliche Weltdeutung in den Rahmenstücken von Johann Rists Monatsgesprächen und ihrer Fortsetzung durch Erasmus Francisci«. In: Flemming Schock (Hg.): Polyhistorismus und Buntschriftstellerei. Populäre Wissensformen und Wissenskultur in der Frühen Neuzeit. Berlin 2012, S. 67-90.

13 Niefanger, Dirk: »Johann Rists Theater-Gespräch von 1666 als wichtige Quelle der barocken Theatergeschichte«. In: Jahn Steiger (Hg.): Johann Rist (1607-1667), S. 185-203. Ganz grundsätzlich zur ParagoneDiskussion Achermann, Eric: »Das Prinzip des Vorrangs. Zur Bedeutung des >Paragone delle arti< für die Entwicklung der Künste«. In: Diskurse der Gelehrtenkultur in der Frühen Neuzeit. Ein Handbuch. Hg. von Herbert Jaumann. Berlin, New York 2011, S. 179-209.
} 
reichlich divergent verhandelt werden, in den Mittelpunkt. ${ }^{14}$ Der hier verfolgte Ansatz will gerade von einer inhaltlichen Ausrichtung abstrahieren und dagegen Fragen nach der formalen Gestaltung der Gespräche und der damit verbundenen Poetik ins Zentrum stellen.

Die These, die im Folgenden verfolgt wird, lautet daher: Die Poetik der Monatsgespräche Johann Rists ist nicht adäquat zu begreifen, wenn man die pragmatische Zielrichtung des Textes außer Acht lässt. Erst in einer pragmatischen Dimension des Textes erschließt sich eine avisierte Sinnebene der Bedeutungszuschreibung, die - sieht man nur auf die inhaltlich-wissenspoetologische oder mediale Zurichtung notwendig defizitär bleiben muss. Gefragt wird demnach nach Formen der Konversation und des Gesprächs, wie sie in lehrreicher Absicht vom barocken Dichter in Szene gesetzt werden und deren wirkungsästhetischen Zwecksetzungen. ${ }^{15}$

\section{Die Monatlichen Unterredungen als Alterswerk - zu Aufbau und Form}

Die Entstehung der Monatsgespräche verdankt sich biographisch klar fassbaren Umständen. Wie Rist den Leser in der Vorrede zum ersten Gespräch wissen lässt, war der Tod seiner langjährigen Ehefrau der Ausgangspunkt der Ausarbeitung der Gespräche. Die Abfassung der Monatsgespräche sollte Trost bergen und den Witwer auf neue, erfreulichere Gedanken bringen. ${ }^{16}$ Der ursprüngliche Plan war auf einen gesamten Jahreszyklus hin angelegt - jeder einzelne Monat sollte mit einem Gespräch bedacht werden. ${ }^{17}$ So viel zum Anlass und avisierten Plan. Der äußere Aufbau der sechs von Rist vor seinem Tod verfassten Gespräche - Januar bis Juni, danach führte Erasmus Francisci die Unterredungen fort ${ }^{18}$ - folgt einem einheitlichen Schema. Den Kern bildet das eigentliche Gespräch zu einem bestimmten Thema, das im Haus des >Rüstigen $<$ - so Rists Pseudonym in der Fruchtbringenden Gesellschaft, deren Mitglied er seit 1647 war $^{19}$ - stattfindet. Geladen sind in der Regel drei weitere Gäste, die ebenfalls einen biographischen Hintergrund in den Sprachgesellschaften der Zeit haben. Sie gehören dem von Rist selbst gegründeten Hamburger

14 Das gilt selbst in Teilen für den mit medialen Fragestellungen befassten Beitrag von Nikola Kaminski.

$15 \mathrm{Zu}$ den Bestandteilen und Merkmalen eines umfassenden Konversationsbegriffs siehe Strosetzki, Christoph: Konversation als Sprachkultur. Elemente einer historischen Kommunikationspragmatik. 2. durchgesehene u. ergänzte Aufl. Berlin 2014, S. 47-147.

16 Rist, Johann: »Das AllerEdelste Nass der gantzen Welt/ Vermittelst eines anmuthigen und erbaulichen Gespräches/ Welches ist dieser Ahrt die Erste/ und zwahr Eine Jänners-Unterredung/ Beschrieben und fürgestellet [1663]«. In: Ders.: Sämtliche Werke. Hg. v. Eberhard Mannack. Bd. 4: Epische Dichtungen (Das allerdelste Naß, Das alleredelste Leben). Berlin/New York 1972, S. 1-120, hier S. 13 f.

$17 \mathrm{Zu}$ den genauen Plänen und deren kontinuierlichem Scheitern siehe Kaminski: »Monatliche Unterredungen, ist ein Journal «.

18 Siehe hierzu Jericke: Johann Rists Monatsgespräche, S. 192-202, sowie Stockhorst: Wissensvermittlung im Dialog, S. 85-90.

19 Vgl. hierzu Conermann, Klaus: Die Mitglieder der Fruchtbringenden Gesellschaft 1617-1650. 527 Biographien. Transkriptionen aller handschriftlichen Eintragungen und Kommentare zu den Abbildungen und Texten im Köthener Gesellschaftsbuch. Weinheim 1985, S. 567. 
Elbschwanenorden (von Rist 1658 gegründet) an. Bisweilen lassen sich die Pseudonyme, unter denen die Gesprächsteilnehmer auftreten, in Klarnamen überführen. Es sind demnach Gespräche unter Gleichen und auch Gleichgesinnten, die in einem grundsätzlich freundschaftlichen Rahmen stattfinden: Gelehrte mit einem weit gefächerten Interessensspektrum. Frauen nehmen nicht an den Gesprächen teil. Das gilt es insofern eigens zu betonen, als es seit Baldessare Castigliones Il Libro del Cortegiano (Das Buch vom Hofmann) ${ }^{20}$ - erstmals 1528 erschienen - zu den immer wiederkehrenden Topoi der theoretisch anleitenden Gesprächsliteratur gehört, dass Frauen einen zivilisierenden und disziplinierenden Einfluss auf die Konversation haben. Aber Rists Gäste wissen sich auch so vorzüglich zu benehmen.

Bevor sich die Diskutanten zu Tisch begeben, um sich dem jeweiligen, zuvor spielerisch verabredeten Thema zu widmen, findet ein erstes Treffen in den beiden Gärten, über die das Haus des Theologen verfügt, statt. Dieser immer gleiche Auftakt bietet Rist einerseits die Möglichkeit, seine LeserInnen ausführlich über Botanik und Arzneikunde zu unterrichten, die Gartengespräche und der Umgang um die Beete erfüllen aber andererseits zugleich die Funktion, bestimmte Konversationsgebote in actu vorzuführen - dazu später mehr. Das ist der eigentliche Zweck der Monatlichen Unterredungen und ihre Poetik dementsprechend daraufhin eingerichtet. Paratextuell flankiert wird die Konversation von Vorreden an den Leser sowie einer Vielzahl der zeittypischen Widmungen an Ehrenpersonen sowie Widmungsgedichte auf den Verfasser und das vorliegende Werk selbst. ${ }^{21}$ Diese hoch konventionalisierten Adressen sind kein bloßes Beiwerk, sondern integraler Bestandteil - auch hier kommen Formen von schriftlich fixierter Höflichkeit und ausgestelltem Anstand zum Tragen. Überdies kommt es mit der Widmung zu einer »symbolischen Übereignung « des Werkes. ${ }^{22}$ Dieser Akt geht also sogar noch deutlich über eine bloße, rein rhetorisch zu verstehende Höflichkeitsbekundung hinaus. Mit den ausgedehnten paratextuellen Bestandteilen dieser Art erreichen die Monatlichen Unterredungen durchaus beträchtliche Umfänge, die einzelnen Bände respektive >Monate< kommen so auf 200 bis 350 Oktavseiten pro Gespräch. Besprochen werden Dinge, die für den allgemeinen Gang der Welt von nicht zu unterschätzender Wichtigkeit sind. Der erste Monat frägt nach dem Alleredelsten Naß der Welt (zur Diskussion stehen Milch, Wasser, Wein und Tinte), im Februar geht es um das Alleredelste Leben (Land-, Soldaten-,

\footnotetext{
${ }^{20}$ Castiglione, Baldesar: Das Buch vom Hofmann (Il Libro del Cortegiano). Übersetzt u. erläutert v. Fritz Baumgart, mit einem Nachwort v. Roger Willemsen. München 1986. Die Hoffrau wird in Buch IV behandelt. Als erster Überblick immer noch unverzichtbar Burke, Peter: Die Geschicke des Hofmann. Zur Wirkung eines Renais-sance-Breviers über angemessenes Verhalten. Übersetzt von Ebba D. Drohlshagen, Berlin 1996.

21 Diese Widmungsgedichte stammen größtenteils aus der Feder von anderen Mitgliedern der Fruchtbringenden Gesellschaft, die dergestalt den Inhalt rekapitulieren und eine Empfehlung für das Werk abgeben. Zu Johann Rists eigenen Widmungsgedichten siehe Arend, Stefanie: »Andreas Tschernig und Johann Rist. Zwei ungleiche Dichter und die Rhetorik ihrer Widmungsgedichte«. In: Jahn Steiger (Hg.): Johann Rist (1607-1667), S. 25-47.

22 Wagenknecht, Christian: [Art.]»Widmung«. In: Reallexikon der deutschen Literaturwissenschaft. Hrsg. v. Jan-Dirk Müller u. a. 3 Bde. Berlin, Boston 2007, Bd. 3, S. 842-845, hier S. 842.
} 
Stadt- und Hofleben), ${ }^{23}$ im März um die Alleredelste Torheit (Suche nach dem Stein der Weisen, Astrologie, die Erfindung eines Perpetuum mobile sowie der Geldverschwendung beim Blumenkauf) ${ }^{24}$; im April-Gespräch geht es um die Alleredelste Belustigung kunst- und tugendliebender Gemüter (die Malerei trägt den Preis davon vor Dramatik, Musik und Poesie) $)^{25}$, das Mai-Gespräch widmet sich der Alleredelsten Erfindung (Lese- und Schreibkunst, Kunst des Mühlenhandwerks, Medizin und Magnetismus ${ }^{26}$ sowie schlussendlich im letzten von Rist selbst verfassten Gespräch zum Monat Juni die Alleredelste Zeitverkürzung diskutiert wird (Todesbetrachtung obsiegt über erbauliche Gespräche, Lektüre, Herstellung von Kunst). ${ }^{27}$ Alle diese Redebeiträge sind enzyklopädisch angelegt; die Behandlung der jeweiligen Themen unterliegt dabei einer vergleichsweise strengen Topik, die eine gewisse Vollständigkeit hinsichtlich der Geschichte, des Gebrauchs, der prominenten Vertreter oder auch nur der Funktionalität garantieren soll. Erschlossen werden so Themen in ihrer Breite und historischen Tiefe. Für den Leser dieser Gespräche heißt das, dass er zukünftig, ausgehend von diesem einmal erworbenen Wissen, diese Kenntnisse erneut geistreich in eigene Konversationen einfließen lassen kann.

Neben diesen inhaltlichen Aspekten sind es allen voran die rhetorischen Fertigkeiten und die zu erlernenden Umgangsformen, die ein gelungenes Gespräch zuallererst ermöglichen. Die Vermittlung dieser Kenntnisse steht mindestens gleichrangig neben den Inhalten, wie Rist nicht müde wird zu betonen. Von zentraler Bedeutung für die Frage nach der Poetik und Pragmatik der hier verfolgten literarischen Kommunikation sind die Vorreden an den Leser, in denen Rist sich an der Explikation seines Programms versucht.

\footnotetext{
23 Rist, Johann: »Das AllerEdelste Leben der gantzen Welt/ Vermittelst eines anmuthigen und erbaulichen Gespräches/ Welches ist dieser Ahrt Die Ander/ und zwahr Eine Hornungs-Unterredung [1663]«. In: Ders.: Sämtliche Werke. Hg. v. Eberhard Mannack. Bd. 4: Epische Dichtungen (Das alleredelste Naß, Das alleredelste Leben). Berlin, New York 1972, S. 121-305.

24 Rist, Johann: »Die AllerEdelste Tohrheit der gantzen Welt/ Vermittelst eines anmuthigen und erbaulichen Gespräches/ Welches ist dieser Ahrt Die Dritte/ und zwahr Eine Märtzens-Unterredung [1664]«. In: Ders.: Sämtliche Werke. Hg. v. Eberhard Mannack. Bd. 5: Epische Dichtungen (Die alleredelste Torheit, Die alleredelste Belustigung). Berlin, New York 1974, S. 1-199.

25 Rist, Johann: »Die AllerEdelste Belustigung Kunst- und Tugendlibender Gemüther/ Vermittelst eines anmuthigen und erbaulichen Gespräches/ Welches ist dieser Ahrt Die Vierte/ und zwahr Eine Aprilens-Unterredung [1666]«. In: Ders.: Sämtliche Werke. Hg. v. Eberhard Mannack. Bd. 5: Epische (Die alleredelste Torheit, Die alleredelste Belustigung). Berlin, New York 1974, S. 200-411.

26 Rist, Johann: »Die AllerEdelste Erfindung der gantzen Welt/ Vermittelst eines anmuthigen und erbaulichen Gespräches/ Welches ist dieser Ahrt Die Fünffte/ und zwahr Eine Mäyens-Unterredung [1667]«. In: Ders.: Sämtliche Werke. Hg. v. Eberhard Mannack. Bd. 6: Epische Dichtungen (Die alleredelste Erfindung, Die alleredelste Zeitverkürzung). Berlin, New York 2015, S. 1-240.

27 Rist, Johann: »Die AllerEdelste Zeitverkürtzung der gantzen Welt/ Vermittelst eines anmuthigen und erbaulichen Gespräches/ Welches ist dieser Ahrt Die Sechste/ und zwahr Eine Brachmonats-Unterredung [1667]«. In: Ders.: Sämtliche Werke. Hg. v. Eberhard Mannack. Bd. 6: Epische Dichtungen (Die alleredelste Erfindung, Die alleredelste Zeitverkürzung). Berlin, New York 2015, S. 241-448.
} 


\section{Die Programmatik der Monatlichen Unterredungen}

Am deutlichsten greifbar wird die Programmatik der Monatlichen Unterredungen in den ersten drei Vorreden an den - »[t]eutschgesinnte[n] «, wie es heißt ${ }^{28}$ - Leser, die deutlich Zielsetzung und Methode reflektieren. Schon diese Leserattribuierung liefert einen ersten Hinweis: Es geht Rist um die Hebung des zivilisatorischen Niveaus seiner deutschsprachigen Zeitgenossen und die Pflege der deutschen Sprache als Literatur- und Wissenschaftssprache insgesamt. ${ }^{29}$ Einer seiner zentralen Einwände gegen die bisherige rhetorische Ausbildung besteht denn auch darin, dass die Kunst des Gesprächs primär Grammatikübungen im Lateinischen umfasse sowie Exempla aus der antiken Literatur bekannt gemacht werden. Doch Rist fällt nicht mit der Tür ins Haus, auch die Vorreden sind - studiert man sie gründlich - bereits selbst eine Anleitung in konversationspraktischer Absicht.

Rist beginnt die Vorrede des ersten Monats mit einer sehr umfassenden captatio benevolaentiae, in der er die Entstehungsumstände der Schrift erläutert. Seine langjährige Ehefrau war nach schwerer und drückender Krankheit verstorben und Rist sucht Trost in der Arbeit. Die Frucht dieser gelehrten, um das Selbst sorgenden Arbeit sind die Monatlichen Unterredungen. Nachdem er den avisierten Aufbau - über die zwölf Monate des Jahres hinweg je ein Gespräch - erklärt hat, kommt Rist zum Zweck, den er sich mit diesem dem Jahresverlauf folgenden Gesprächszyklus gesetzt hat. Ganz der Horazschen Maxime entsprechend sollen die Gespräche sowohl $>$ nützen $<$ als auch >erfreuen<. Nur in dieser Kombination des prodesse et delectare lässt sich die verfolgte Absicht erreichen. Die gesamte Programmatik kulminiert in einem einzigen - mit zunehmender Länge - unübersichtlichen Satz. Mitglieder des von Rist neu gegründeten Elbschwanenordens sind zugegen

[...] als Unterredner / oder Zusammensprächer sollten ingeführet werden / welches den vielen verständigen / sonderlich aber der Kunst- und Tugendlibenden Jugend / nicht nur anmuthig und lustig / sondern auch sehr nützlich würde sein zu lesen und zugebrauchen / zumahlen sie bisweilen solche Sachen in mehrerwähneten Gesprächen werden finden / derogleichen ihnen sonst wenig fürkommen / demnach selbige nicht so viel aus den Büchern / als aus eigener Erfahrung der rechten und wahren Lehrmeisterinn aller Dinge sind genommen. ${ }^{30}$

Rist bringt hier gleich mehrere Punkte zur Sprache, die für die Frage nach gelingender Konversation von Bedeutung sind. Es werden einerseits Erfahrung und Bücherwissen zueinander ins Verhältnis gesetzt und zugleich damit das Verhältnis von Lektüre und Gespräch in ihrem dezidierten Nutzen für die Ausbildung der Jugend. Denn gerade diese ist die primäre Zielgruppe der Ristschen Gespräche. Sie zielt auf eine umfassende rhetorische Schulung und dient der Bereitstellung eines Wissensreservoirs, aus dem für künftige Unterhaltungen geschöpft werden kann -

\footnotetext{
28 Rist: Das alleredelste Naß, S. 13.

29 Siehe auch Mast, Thomas: »Patriotism and the Promotion of German Language and Culture. Johann Rist's Rettung der Edlen Teutschen Hauptsprache (1642) and the Language Movement of the Seventeenth Century.« In: Daphnis 30 (2001), S. 71-96.

30 Rist: Das alleredelste Naß, S. $15 \mathrm{f}$.
} 
auch eine Übersetzung des Gesprächs ins Lateinische im Sinne einer Stilübung wäre denkbar und hilfreich, wie Rist meint. ${ }^{31}$ Zum Ende der Vorrede wird >Der Rüstige< noch einmal explizit in seiner Programmatik:

Jch will hie nicht sagen / wie die Jugend die Teütsche Redekunst aus solchen und dergleichen Schrifften / mit sonderbarer Beliebung erlernen und fast von allen Dingen / so unter dem Himmel gefunden werden / ein libliches und vernünftiges Gespräch gestalten / dadurch also gleichsahm mit Spielen der wahren Geschiklichkeit könnten teilhaft werden. ${ }^{32}$

Der entscheidende Punkt, auf den es Rist ankommt, ist die Kombination rhetorischer Fertigkeiten verbunden mit einem mannigfaltigen Alltags- oder Weltwissen in aktueller Perspektive. Denn wie er in einer weiteren Vorrede ausführt, kann das die klassische Rhetorikausbildung, die sich an den kanonischen Texten der Alten orientiert, nicht leisten. Selbst wenn man die Konversationsregeln dergestalt erlernt hat, fehlt einem der Redegegenstand oder das Wissen um aktuelle Entwicklungen im gelehrten Feld. Mit einem Terenz-Zitat, so Rist, kann man dann nicht wirklich punkten. Man bleibt ein uninteressanter Gesprächspartner, der inhaltlich nichts Substanzielles beizutragen hat. Aus dieser Einsicht resultiert die Deutschsprachigkeit der Texte ebenso wie ihr dezidierter Weltbezug.

Rist will seine Gespräche ferner explizit als »Spiel« begriffen wissen, womit er sich einerseits in die Tradition von Georg Philipp Harsdörffers Frauen-Zimmer-Gesprächsspielen stellt, ${ }^{33}$ zugleich aber auch auf einen methodischen Aspekt anspielt, wie mir scheint. Denn das Wesen eines Spiels ist ja zunächst einmal, sich an gewisse Regeln zu halten. Sie bilden den Rahmen des Spiels, innerhalb dessen man sich allerdings vergleichsweise frei bewegen kann. Diese Spiel-Metaphorik trifft den Charakter eines gelungenen Gesprächs schon ziemlich präzise. Alle können ihre je eigenen Fertigkeiten und Kenntnisse einbringen. Die Fertigkeiten beziehen sich auf die Verfügbarkeit der (rhetorischen) Regeln, die Kenntnisse auf die Gesprächsinhalte selbst. Beides ist notwendig für das Gelingen eines Spiels.

Die weiteren Vorreden wiederholen, vertiefen und variieren diese Grundprogrammatik, wie sie in der Vorrede zum Januar-Gespräch vorgestellt wird. Zudem setzten sie je eigene Schwerpunkte, die weitere Perspektiven auf den Nutzen der Texte eröffnen. Neben der schon ab dem zweiten Band wiederkehrenden Entschuldigung für die Verzögerung des Erscheinens rücken etwa Formen der Kritik und die Rolle des

\footnotetext{
$31 »[\ldots .$.$] wen sie nemlich diselben [die Gespräche, M.M.] in die Lateinische Sprache übersetzeten / da sie$ nicht nur beider Sprachen mächtig / sondern auch aller darin enthaltenen Sachen / mit Lust / ja fast ohne alle Mühe kund und fähig werden / und also einen gedoppelten Nutzen daraus haben und ziehen können.« Ebd, S. $15 \mathrm{f}$.

32 Ebd., S. 17.

33 Grundlegend Zeller, Rosmarie: Spiel und Konversation im Barock. Untersuchungen zu Harsdörffers Gesprächspielen. Berlin 1974; ferner Doms, Misia Sophia: »Der Leser als Gesprächsthema und Dialogpartner in Georg Philipp Harsdörffers >Frauenzimmer Gesprächspielen< (1641-1649) und Christian Thomasius' >Monatsgesprächen<(1688-1689). In: Zeitschrift für Germanistik. NF 23, H. 3 (2013), S. 510-523.

34 Das Alleredelste Leben, S. $141 \mathrm{f}$.

35 Rist: Die Alleredelste Torheit, S. 16.
} 
Kritikers, ${ }^{34}$ die lebenslange Nützlichkeit der Rhetorik ${ }^{35}$ oder die paragone delle arti neben anderem in den Blick.

\section{Rhetorik - Gelehrsamkeit - Konversation}

Die Fokussierung auf eine Konversationslehre aus dem Geist der Rhetorik ist in allen Vorreden deutlich spürbar. Denn das Gespräch - oder genauer: die Form des Gesprächs - ist weitaus mehr als ein auf Geselligkeit angelegtes Unterfangen. In der Vorrede zum Hornungs-Gespräch (i.e. Februar) kommt Rist auf das Verhältnis von Rhetorik und Wahrheit zu sprechen. Nachdem die Frage zum Stellenwert von Erfahrungswissen generell diskutiert wurde und dergestalt eruiert werden sollte, was eigentlich der (Buch-)Gelehrte vom Leben wissen kann - »da er doch immer über den Büchern liget / Bücher liset / Bücher schreibet / und lässet sein Gesinde die Haushaltung verwalten $\ll^{36}-$, kommt Rist auf die Macht der Rhetorik zurück. Denn,

[...] das [sic, passim] man durch die Redekunst / auch wol scheltenswürdigen Dingen ein Färblein könne anstreichen / und einem heslichem Leibe gar einen schönen Mantel ümhängen / unterdessen wird kein Mensch leugnen können / das unter den Kriegsleuten und Soldaten / ja wohl Gottesfürchtige / ehrliche / Gewissenhaffte und Tugendlibende Leute / [...]. ${ }^{37}$

Rhetorik beinhaltet insonderheit also auch die Darstellung des Falschen als wahr und des Wahren als falsch. In der Rhetorik geht es nach Rist nicht primär um Wissen und Wahrheit - das ist der Rhetorik äußerlich und kommt erst in einem zweiten Schritt hinzu - sondern es geht darum, sein eigenes Anliegen geschickt zu argumentieren. Dass es sich hierbei um ein Rollensprechen handelt - der Sprecher also nicht unmittelbar auf seine Aussagen verpflichtet werden kann - versteht sich für Rist von selbst: »So darf man darum nicht flugs den Verfasser schelten / oder ihn einiger Parteiligkeit beschüldigen / sonderlich / wen man sein rechtes Absehen nicht verstehet. « ${ }^{38}$ Diese Haltungen und die dazugehörige Technik einzuüben, dienen die von ihm verfassten Gespräche. Denn dass bei aller Künstlichkeit der Gespräche die Kunstfertigkeit nicht sichtbar werden soll, ${ }^{39}$ ist gerade der Ziel- und Endpunkt seiner Demonstrationen: »die grösseste Kunst eines gelehrten Mannes ist / die Kunst können verbergen. $\aleph^{40}$ Gelingen kann dies nur vor einem aktuellen Hintergrund, der mit der antiken Rhetorik nicht zu erreichen ist:

\footnotetext{
36 Rist: Das Alleredelste Leben, S. 143.

37 Ebd., S. 144.

38 Ebd.

39 Grundlegend für diesen Zusammenhang Geitner, Ursula: Die Sprache der Verstellung. Studien zum rhetorischen und anthropologischen Wissen im 17. und 18. Jahrhundert. Tübingen 1992.

40 Rist: Das alleredelste Leben, S. 145. Und in der Vorrede des Märzgespräches heißt es weiter: »Jch suche ja durch herausgebung solcher Bücher und Schrifften / weder Ehr noch Ruhm zu erlangen; Nur hab ich allen Kunstliebenden / zuforderst aber jungen Leüten und Studenten in denselben zeigen wollen / wie man von allerhand Künsten und Wissenschaften / bald guthes / bald böses könne fürbringen / und / vermittelst der Redekunst / von derselben vielerhand schöne und nützliche Gespräche anstellen.« Rist: Die Alleredelste Torheit, S. $13 \mathrm{f}$.
} 
Es ist ja in Wahrheit hoch zu beklagen / das man die libe Jugend so viele Jahre mit den Grammaticalischen Possen martert und plaget / und / wenn man offt grosse und erwachsene Jüngelinge fraget / was man ihnen doch für Bücher oder Authores in den Schuhlen habe fürgelesen und erklähret? So ist es gemeinlich der Terentius nebst dem Ovidius, und / wen sie gahr hoch gestiegen sind / auch wol der Plautus. ${ }^{41}$

Was lernt man daraus konkret? - eben »so viel Latein [...] / das er es zur Noth kann reden und schreiben « ${ }^{42}$ Damit ist für einen jungen Mann, der sich auch außerhalb der engen akademischen Kreise bewegen möchte, hinsichtlich seiner Fähigkeit zur Konversation wenig gewonnen:

Höhren sie / das man bald von diesen / bald von jennen schönen und nützlichen Wissenschafften eine Unterredung anstellet / so sitzen sie da / wie die unvernünfftigen Rinder und Schöüsen / sperren Nase und Maul weit auff / höhren es zwahr an mit Verwunderung / wissen aber / wie andere / das allergeringste dazu nich zu sagen / oder derogleichen etwas fürzubringen. ${ }^{43}$

Die Jugend ist also ohne eine fundierte rhetorische Ausbildung mit gleichzeitigem Wissenserwerb in den relevanten Wissenschaften nicht konversationsfähig. Das ist genau jene Form der Unbeholfenheit und Sprachlosigkeit gegenüber höher gestellten Standespersonen, für die sich im 18. Jahrhundert der Begriff der >Blödigkeit< durchsetzen wird. ${ }^{44}$ Wird das im 18. Jahrhundert zu einem fundamentalen Problem des Individuums, so ist der Misere hier bei Rist noch deutlich einfacher Abhilfe zu schaffen. Nämlich schlicht durch eine richtige Ausbildung - also die Kenntnis der Regeln guter Konversation -, gelingenden Beispielen und schlussendlich eigener Übung. Diesen Dreischritt aus praeceptum (Regel), exemplum (Beispiel) und exercitio (Übung) kennt man auch aus den Poetiken der Zeit. ${ }^{45}$ Es ist das gleiche Vorgehen, mit dem Dichtung entstehen kann. Rist ist sich - in der Nachfolge von Martin Opitz, dessen Programm er eifrigst propagiert - dieser Methodik vollauf bewusst und überträgt sie aus dem Bereich der Dichtung auf die Ausbildung zur Konversation. Die literarische Gattung ist hier selbstredend das Gespräch, das hier gleich in einer doppelten Funktion auftritt. Es ist zugleich als das gute Beispiel, das exemplum zu sehen, das aber gleichermaßen - im Umkehrschluss - die Möglichkeit bietet, für sich selbst gewisse Regeln zu extrahieren, die fernerhin Gültigkeit haben. Rist umgeht es unter der Aufrufung des Spielcharakters der Gespräche, fixe Regeln als ein kodifiziertes Regelwerk zu formulieren, und dennoch liefern die Monatlichen Unterredungen diese bereits in praktisch angewandter Form. Bildlich gesprochen handelt es sich gerade nicht um eine ausformulierte, dafür aber um eine implizite Poetik, die eben in der literarischen Kommunikation ihre Gestalt gewinnt.

\footnotetext{
41 Rist: Die Alleredelste Torheit., S. 14.

42 Ebd.

43 Ebd., S. 14 f.

44 Siehe hierzu umfassend Stanitzek, Georg: Blödigkeit. Beschreibungen des Individuums im 18. Jahrhundert. Tübingen 1989.

45 Vgl. Knape, Joachim: Poetik und Rhetorik in Deutschland 1300-1700. Wiesbaden 2006, S. 48-59.
} 
Für den Leser bleibt daher die Frage im Raum, was sich aus diesen Gesprächen für eine gelungene Konversation lernen lässt?

Das Angebot, das Rist hier liefert, ist in dieser Hinsicht äußerst reichhaltig. Erlernbar ist in erster Linie einmal eine Praxis der Topik (aufgefächert nach Ursprung, Gebrauch, Notwendigkeit und Wert sowie Vortrefflichkeit) ${ }^{46}$, also die Art und Weise, wie man sich einen Gegenstand mittels präzise gestellter Fragen in systematischer Weise erschließt (das, was die klassische Rhetorik unter dem Begriff der inventio subsumiert und nur theoretisch ausformuliert). Ferner differenzierte Formen der Höflichkeit je nach Gesprächsstadium (etwa das Eingangskompliment für das Gegenüber zum Einstieg in das Gespräch), Bescheidenheitsgesten in der Nachfrage und Präzisierung, Fragen nach der Reihenfolge der Sprecher und des Vortritts bzw. Vorzugs der Redner, ferner unterschiedliche Arten des Gesprächseinstiegs, Überleitungen, Repliken und das elegante Abschließen eines Sujets. Thematisiert werden aber auch dem Gespräch und dessen Verlauf nur vermeintlich >äußere<Umstände wie eine passende Sitzordnung der Teilnehmer, die der Unterhaltung dienlich ist, die Räumlichkeiten, in denen das Gespräch stattfindet (Garten und Haus) oder die Alimentation mit Speis' und Trank (Wein ja - unbedingt, aber auch nicht zu viel). Konversation wird hier nicht nur als ein sprachlicher Akt begriffen, sondern in seiner vollumfänglichen Extension als Lebensereignis ausgestellt und thematisiert.

Diese von Rist vorgestellten Gedanken sind freilich keineswegs neu oder gar originell, ganz im Gegenteil - sie fußen auf der langen Tradition der Rhetorik seit der Antike. Unter Aufbietung zahlreicher lebensweltlich-praktischer Anreicherungen gelingt es ihm mit der Form des dergestalt dargebotenen Gesprächs - zumal in deutscher Sprache - ein Novum hinsichtlich der Didaktik der Konversation zu schaffen und damit der Blödigkeit der Jugend seiner Zeit entgegen zu arbeiten. Der sich in tiefer Trauer befindliche Theologe Rist demonstriert die Bedingungen der Möglichkeit guter, d.h. gelingender Konversation, indem er uns Blaupausen gelungener Gespräche vor Augen führt. Darin besteht die ganze Raffinesse dieser unter gattungspoetischen Gesichtspunkten bisher schwer greifbaren Texte. Erst die Überblendung beider Ebenen - Theorie und Praxis in Simultanität - erweist ihre volle Wirksamkeit und Zweckmäßigkeit.

\section{Gesprächsliteratur und >Konversationsöffentlichkeit $<-$ Skizze eines Forschungsfeldes}

Die eingangs gestellte Frage nach dem gattungssystematischen Ort der Monatlichen Unterredungen - wie von Alfred Jericke angemahnt und eingefordert - blieb bislang unbeantwortet. Das Unbehagen einer eindeutigen Zuordnung in der Forschung hat sich seit den 1920er Jahren kaum gelegt, wenngleich die theoretischen Angebote der Zahl nach freilich zugenommen haben. Der in diesem vorliegenden Fall in der germanistischen Literaturwissenschaft am breitesten rezipierte und auch sicherlich wirkmächtigste Vorschlag geht auf Jürgen Habermas und dessen Strukturwandel der Öffentlichkeit (erstmals 1962) zurück. Die dort konstatierte Entstehung einer

\footnotetext{
46 Siehe hierzu etwa Rist: Das alleredelste Naß, S. 96.
} 
»bürgerlichen Öffentlichkeit«, die eine vormals herrschende »repräsentative Öffentlichkeit« mit dem Ausgang des 17. Jahrhunderts und beginnenden 18. Jahrhunderts abgelöst habe, strukturiert nach wie vor - mit vielerlei Einspruch im Detail - die Wahrnehmung periodisch angelegter Literaturformen wie der hier vorgestellten. Dieser sozialgeschichtliche Zugang schleift sich nur ganz allmählich ab. So werden eine Vielzahl von Texten des 17. Jahrhunderts zu defizitären Vorläufern einer periodischen Presse des 18. Jahrhunderts - allen voran der Moralischen Wochenschriften - erklärt. Aber nicht nur die späten Werke Rists fallen in die Latenzphase dieser Umstellung, vielmehr ist Gesprächsliteratur in den Jahrzehnten zwischen 1640 und 1710 ein beinahe omnipräsentes Genre: Georg Philipp Harsdörffers FrauenzimmerGesprächspiele (1641-1649) reihen sich zu weiteren Projekten >barocker $>$ Provenienz, etwa von Philipp von Zesens Rosenmând (1651) und Siegmund von Birkens Pegnesische Gesprächspiel-Gesellschaft (1665). Erasmus Franciscis Monatsgespräche (1663-1671) bringen das Ristsche Vorhaben zu einem Ende, mit Christian Thomasius' Monatsgesprächen (1689) wird $>$ Kritik $<$ neu gedacht, ${ }^{47} \mathrm{ihm}$ folgen Tentzels Monatliche Unterredungen (1689--1698); die Reihe verläuft weiter über Nikolaus Hieronymus Gundlings Neue Unterredungen (1702) bis zu Sinolds Das neue und curiöse Caffée-Hauß (1707-1708), um nur die prominentesten Vertreter zu nennen. All diese Texte seien Vertreter einer frühen Formationsphase einer Zeitschriftenkultur und damit einer >bürgerlichen Öffentlichkeit< im Habermas'schen Sinn avant la lettre. So richtig aufgehen und einleuchten wollte diese >Vorläuferschaft $<$ häufig nicht, gleichwohl hielt man an ihr fest. Dabei krankt das Konstrukt schon an einer logischen Inkohärenz: All die Beteiligten verwirklichten ihre Werke nicht unter der Ägide einer kommenden > Öffentlichkeit<, von der sie selbst noch nichts wussten oder gar wissen konnten. Die Marginalisierung der hier nur in Auswahl genannten Schriften im Sinne eines noch nicht vollständig zu sich gekommenen Schreibens unter den Bedingungen einer erst noch im Entstehen begriffenen >bürgerlichen Öffentlichkeit< offenbart die teleologische Grundausrichtung der Forschung. Die naheliegende Frage, die sich mithin stellt, ist eine ganz andere, selbst wenn man an der Diagnose festhält. Gesetzt den Fall, dass die hier zur Diskussion stehenden Schriften weder vollumfänglich in der Terminologie einer >repräsentativen Öffentlichkeit< beschrieben werden können, noch genugsam die Kriterien einer >bürgerlichen Öffentlichkeit< erfüllen, so handelt es sich offensichtlich um ein Drittes, das bisher nicht benannt wurde.

Systemtheoretisch gesprochen und an der Terminologie Niklas Luhmanns orientiert, könnte man diese historische Umbruchphase - um 1700 - als zu Ende gehende Phase einer stratifikatorisch verfassten Gesellschaft hin zu einer sich langsam aber stetig funktional ausdifferenzierenden Gesellschaft beschreiben, die auch dem Gelehrten einen nicht eindeutig festschreibbaren Status zwischen den gesellschaftlichen Gruppierungen zukommen lässt. ${ }^{48}$

\footnotetext{
47 Jaumann, Herbert: Critica. Untersuchungen zur Geschichte der Literaturkritik zwischen Quintilian und Thomasius. Leiden 1995.

48 Füssel, Marian: Gelehrtenkultur als symbolische Praxis. Rang, Ritual und Konflikt an der Universität der Frühen Neuzeit. Darmstadt 2006.
} 
Was Habermas zur Zeit der Abfassung seiner Habilitationsschrift nicht sehen konnte - schlicht weil ihm die Terminologie dazu fehlte, ${ }^{49}$ ist die mediale Verfasstheit seiner eigenen Begriffe von Öffentlichkeit, ${ }^{50}$ der ein passendes Bindeglied fehlt. Ich schlage daher vor, dass man als Zwischenglied eine Phase der >Konversationsöffentlichkeit< annimmt, die an den beiden Formen von Öffentlichkeit partizipiert, die Jürgen Habermas in Anschlag gebracht hat, indes nicht vollständig in ihnen aufgeht. Zwar bedienen sich Schriften, die hier zur Gesprächsliteratur gezählt werden, Formen repräsentativer Öffentlichkeit, aber sie bilden diese Art von Öffentlichkeit nicht länger direkt ab. Denn »[diese] repräsentative Öffentlichkeit konstituiert sich nicht als ein sozialer Bereich, als eine Sphäre der Öffentlichkeit, vielmehr ist sie, [...] so etwas wie ein Statusmerkmal. $\ll^{51}$ Die Medialität der Gesprächsliteratur ist bereits eine andere.

Heinrich Bosse legt den Finger in die Wunde: »Die räsonierenden Bürger stattet Habermas mit Besitz und Bildung aus, mit Besitz von Seiten der Wirtschaftsgeschichte, mit Bildung allerdings aus dem Nichts. $\ll^{52}$ Der blinde Fleck bei Habermas, den Bosse hier anspricht, würde ich in den Texten und Praktiken dessen verorten, was ich >Konversationsöffentlichkeit< nenne. Was in England die Gentlemenclubs und in Frankreich die Salons sind - für die es beide im deutschsprachigen Raum keine wirklichen Äquivalente gibt - sind vielleicht genau jene realen und textuellen Gesprächszirkel, wie sie Johann Rist und seine Nachfolger vorstellen. Teil einer >bürgerlichen Öffentlichkeit< sind sie damit nicht unbedingt. Denn Rists Monatliche Unterredungen zielen nicht auf Nachrichten ab, was sie der Sphäre einer bürgerlichen Öffentlichkeit annähern würde, sondern sie bieten inhaltlich eine aktualisierte Form von Wissen aus ganz unterschiedlichen Gebieten auf dem Stand der Zeit, die allerdings bereits auf ein bestimmtes Publikum zielt. Formal betrachtet und ihrer poetologischen Absicht folgend handelt es sich um eine Anleitung zu gelungener Konversation, die zu einem erklecklichen Maß - aber eben auch nicht nur - aus Repräsentation besteht. Unter dem Begriff der >Konversationsöffentlichkeit< ließe sich dann jener Bereich gesellschaftlichen Lebens verstehen, der weder strikt privat, noch ausschließlich öffentlich ist, sondern einer Form von Repräsentation dient, deren Regeln höfischen Ursprungs sind und die insofern modifiziert und verfeinert werden, als sie den strengen protokollarischen Anforderungen des Hofes nicht mehr genügen müssen. Erst auf dieser Grundlage kann Konversation als Spiel begriffen werden, das zwar einen dezidierten Nutzen verfolgt, aber das nicht unter dem Damoklesschwert eines endgültigen Ausschlusses aus der (höfischen) Gesellschaft steht. Der Spielcharakter entlastet von einer existenziellen Bedrohung. Die Gesprächsliteratur der zweiten Hälfte des 17. Jahrhunderts trägt diesem Umbruch Rechnung, indem sie versucht, genau jene Fertigkeiten zu vermitteln, die in sich neu ergebe-

\footnotetext{
49 Aber genau das ist der Status der Rezeptionssituation der literaturgeschichtlichen Adaptionen.

50 Sowohl >repräsentative< als auch >bürgerliche< Öffentlichkeit konstituieren sich in einem Kommunikationsverhältnis, mithin also medial.

51 Habermas, Jürgen: Strukturwandel der Öffentlichkeit. 6. Aufl. Neuwied, Berlin 1974, S. 19 f.

52 Bosse, Heinrich: »Öffentlichkeit im 18. Jahrhundert. Habermas revisited.« In: Navigationen - Zeitschrift für Medien- und Kulturwissenschaften, Jg. 15 (2015), Nr. 1, S. 81-97, hier S. 81.
} 


\section{nen gesellschaftlichen Konstellationen von Nöten sind, um in der Konversation zu} reüssieren.

Funding Open Access funding provided by Projekt DEAL.

Open Access Dieser Artikel wird unter der Creative Commons Namensnennung 4.0 International Lizenz veröffentlicht, welche die Nutzung, Vervielfältigung, Bearbeitung, Verbreitung und Wiedergabe in jeglichem Medium und Format erlaubt, sofern Sie den/die ursprünglichen Autor(en) und die Quelle ordnungsgemäß nennen, einen Link zur Creative Commons Lizenz beifügen und angeben, ob Änderungen vorgenommen wurden.

Die in diesem Artikel enthaltenen Bilder und sonstiges Drittmaterial unterliegen ebenfalls der genannten Creative Commons Lizenz, sofern sich aus der Abbildungslegende nichts anderes ergibt. Sofern das betreffende Material nicht unter der genannten Creative Commons Lizenz steht und die betreffende Handlung nicht nach gesetzlichen Vorschriften erlaubt ist, ist für die oben aufgeführten Weiterverwendungen des Materials die Einwilligung des jeweiligen Rechteinhabers einzuholen.

Weitere Details zur Lizenz entnehmen Sie bitte der Lizenzinformation auf http://creativecommons.org/ licenses/by/4.0/deed.de. 